\title{
Randomized, Cross-Over Evaluation of Mobile Phone vs Paper Diary in Subjects with Mild to Moderate Persistent Asthma
}

\author{
Eli O. Meltzer ${ }^{*}$, , Norma Kelley ${ }^{2}$ and Melbourne F. Hovell ${ }^{2}$ \\ ${ }^{1}$ Allergy \& Asthma Medical Group \& Research Center, San Diego, CA, USA \\ ${ }^{2}$ Center for Behavioral Epidemiology and Community Health, Graduate School of Public Health, San Diego State Uni- \\ versity, San Diego, CA, USA
}

\begin{abstract}
Diaries are frequently used to evaluate therapy. Forgetfulness, however, can lead to missed entries. With paper diaries, these missing entries can be backfilled, compromising the reasons for using a diary. Electronic diaries are a potential means of mitigating this limitation. The pilot study was conducted to evaluate use of a mobile phone diary. Twelve subjects with mild persistent asthma were randomly assigned to mobile or paper diary for 2 weeks and then crossed over to use the other diary type for next 2 weeks. Of the 12 subjects, 7 preferred the mobile diary. However, the mean prevalence of missing data was greater when using the mobile $(18 \% \pm 9 \%)$ compared to paper diary $(9 \% \pm 4 \% ; P=0.05)$. In conclusion, the mobile diary was preferred by slightly more subjects. The greater prevalence of missing data when using this diary most likely results from the inability to backfill missing entries.
\end{abstract}

Trial Registration: Clintrials.gov NCT00367263 (http://clinicaltrials.gov/ct2/show/NCT00367263).

\section{INTRODUCTION}

Recent guidelines recommend controller therapy in the management of patients with persistent asthma and state that an ICS is the most effective controller currently available [1, 2]. However, whether meaningful differences exist between the various ICSs that have been approved for clinical use remains unresolved. For example, ciclesonide, delivered as a solution in a HFA MDI (Alvesco ${ }^{\circledR}$, Sanofi-Aventis U.S., Bridgewater, NJ; Nycomed/Altana Pharma U.S., Florham Park, NJ.) has been found to have superior safety and/or efficacy than older ICS in some trials [3-5] but not in others [68].

Evaluating individual ICSs frequently requires collecting data from outpatients on a daily basis over a prolonged period of time. Typically, a written patient diary is employed for this collection [8-11]. However, the effectiveness of this collection is often limited by noncompliance of study subjects with the prescribed protocol for diary entries [12]. Lack of diligence, motivation and simple forgetfulness, however, can lead to missed diary entries; moreover, these missed entries are not always recognized as such since embarrassment and/or lack of understanding promotes backfilling of these entries prior to returning the diary to the investigator [13]. Even when performed in good faith, this backfilling defeats the purpose of using a diary to minimize the effects of recall and subsequent events on outcomes [13].

More recently, use of electronic diaries has been recommended as a potential means of overcoming this limitation [13-16]. These electronic diaries can encourage real-time compliance through the use of interactive features, such as

*Address correspondence to this author at the Allergy \& Asthma Medical Group \& Research Center, San Diego, CA 92123, USA;

E-mail: eomeltzer@aol.com signaling the subject when assessments are due, permitting responses only during particular windows of time, and time stamping these responses [13]. Acceptance of these electronic systems, however, can vary depending upon their engineering requirements. Devices vary in their requirements for downloading data. Some store diary entries which are downloaded at the study site. Others have the subject transmit their entries directly to a server via an analogue or digital phone line. These types of phone connections are becoming less prevalent with cell phones far surpassing the number of wired connections worldwide [17]. Therefore, a wireless system would be advantageous. In addition, overall ease of use and subject familiarity/comfort with similar electronic devices should enhance acceptance of these electronic systems [14].

The VOCEL ${ }^{\circledR}$ Mobile Diary is a new mobile phone-based system that can capture and transmit study data. It is lightweight, portable, and adaptable to the needs of a particular trial. It can remind subjects when it's time to take study medication and/or enter their report and can then time stamp this report. Use of this dairy system in a clinical trial has not been previously evaluated. The objective of this pilot evaluation was to assess use and patient acceptance of the VOCEL ${ }^{\circledR}$ Mobile Diary in comparison with standard paper reporting methods in documenting the efficacy, safety, compliance, and convenience of inhaled ciclesonide in patients with mild to moderate persistent asthma. Secondarily, we documented effect sizes and explored the statistical significance of larger observed effects.

\section{MATERIALS AND METHODOLOGY}

This was a single center, randomized, cross-over evaluation of subjects with mild to moderate persistent asthma. Following a screening assessment which included medical history, physical examination, and pulmonary function testing, subjects were issued a traditional paper (Fig. 1) or VO- 
Subject Initials

Protocol Number

SUBJECT DIARY CARD

Subject Number

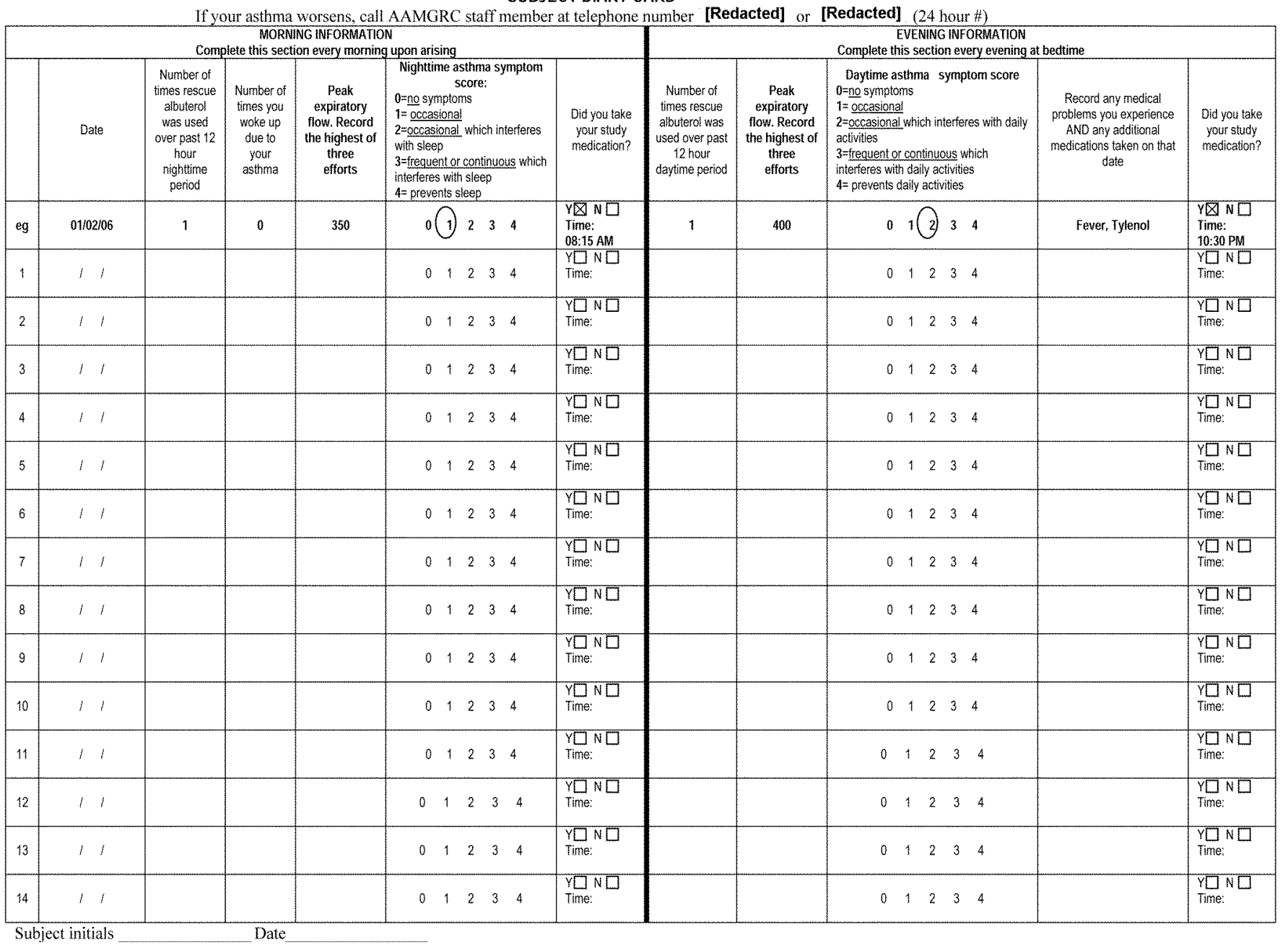

Fig. (1). Traditional paper diary used in the current evaluation.

CEL ${ }^{\circledR}$ Mobile Diary (Fig. 2) in random order and instructed to complete this diary twice daily for a period of 2 weeks. In addition, subjects randomized to the mobile phone diary received an electronic alert from the device at each scheduled time for diary entry throughout the evaluation period. At the end of this period ( \pm 2 days), subjects returned their diary, had their pulmonary functions assessed, and were issued the other diary to use for the next 2 weeks. Subjects then returned the second diary, had their pulmonary functions reassessed, and answered questions regarding both diary type preference and study medication (see Appendix 1 for exact questions).

Subjects currently taking an ICS were instructed to refrain from using this agent for the duration of the study. All subjects were given a ciclesonide MDI (40 $\mu \mathrm{g} / \mathrm{puff})$ as controller therapy and instructed to take 2 puffs twice daily (total daily dose $160 \mu \mathrm{g}$ ciclesonide) throughout the study. This change in prescription mimics conditions likely when testing a new medication or a medication new to the patient. In addition, all subjects were given an albuterol MDI as rescue medication to be used on an as needed basis throughout the study. Finally, all subjects were given a PIKO-1 ${ }^{\mathrm{TM}}$ (Pulmonary Data Services, Inc., Louisville, CO) peak flow meter and instructed in its use. Subjects were told to measure their PEFR 3 consecutive times just before their morning and evening ciclesonide dose and to record the greatest of these 3 measurements in their diary.

\section{Study Population}

The study population consisted of male and female subjects $\geq 12$ years of age with mild to moderate persistent asthma, as defined by the National Heart, Lung, and Blood Institute [18], for at least 6 months. Eligible subjects included patients who had received no ICS therapy, ICS monotherapy, or ICS in combination with a long acting beta agonist in the 30 days prior to screening. Subjects were excluded from study participation if they had a respiratory tract infection in the preceding 14 days; had active or quiescent tuberculosis; had a history of chronic bronchitis, chronic obstructive pulmonary disease, or emphysema; smoked tobacco in the preceding 6 months or had a lifetime 10 pack-year smoking history; had any other serious concomitant disease or condition that might interfere with study completion or interpretation; had received an investigational drug within the past 30 days; were pregnant or trying to become pregnant; or were breast feeding. 
A)



C)

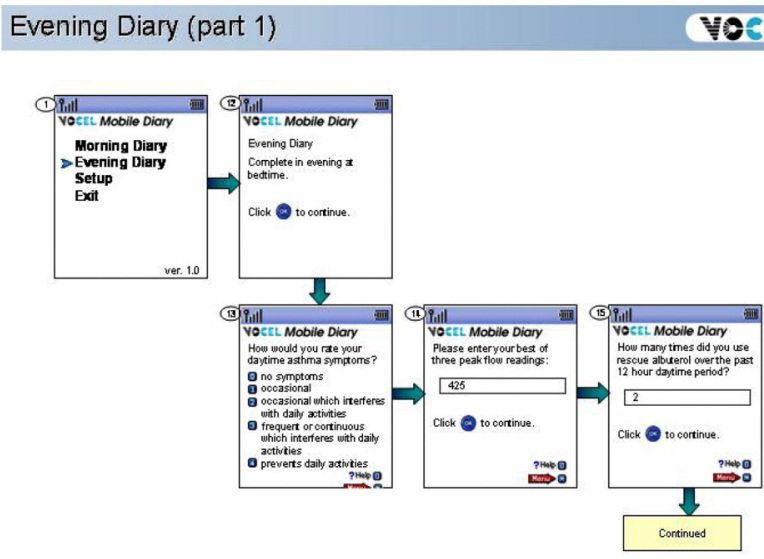

Preen Penting

E)

Evening Diary (Medical Problem Entry)

WOCEL'



B)



D)

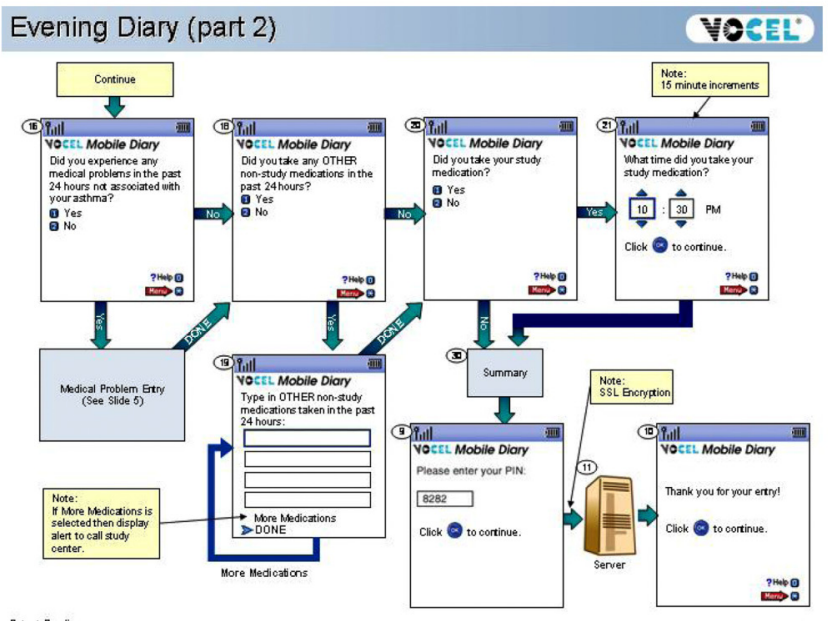

F)

Mobile Diary

YOCEL'

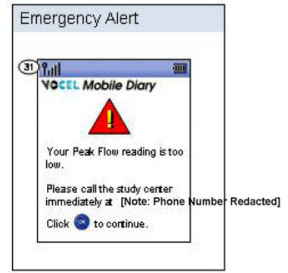

Fig. (2). Screens (A through F) of the VOCEL ${ }^{\circledR}$ Mobile Diary as configured for the current evaluation. 
The study protocol was approved by the Sterling Institutional Review Board and all subjects provided written informed consent prior to participation in any study specific procedures.

\section{Outcome Measures}

The primary outcome measure was the proportion of subjects who preferred using the VOCEL ${ }^{\circledR}$ Mobile Diary vs the traditional paper diary.

Secondary outcome measures included 1) influence of diary type on drug assessment as reflected by recorded compliance, use of rescue medication, PEFRs, asthma symptom scores, and nocturnal awakenings and 2) ciclesonide efficacy and safety as reflected by both diary entries and objective assessment of pulmonary function at the end of each diary period.

\section{Statistical Analysis}

As a pilot evaluation, this study was not designed to detect group differences across the variables analyzed and a formal power analysis was not performed prior to study initiation. Data were inspected concerning distributional assumptions for the statistical tests to be employed. It is common for small samples to result in skewed distributions. For this study, all analyses were computed with both parametric and nonparametric analyses. Parametric analyses were reported for ease of interpretation. Participants were not moved from one condition to the other based on evidence of exposure to the "wrong" condition. In this sense, an intent-to-treat analysis was employed. However, missing data were ignored and imputation procedures were not used. All analyses were computed using standard statistical program packages (SPSS).

Subjects functioned as their own control with pairwise tests used to compare subjects after each diary period. Data were analyzed using descriptive statistics and general linear models (GLM) to test for differences between and within conditions with $P \leq 0.05$ used to indicate statistical significance. These parametric analyses were confirmed using the Wilcoxon nonparametric test. Significance is reported for either the Wilcoxon or the GLM test. Unless otherwise indicated, data are reported as mean \pm standard deviation.

\section{RESULTS}

\section{Study Population}

Thirteen potential subjects were screened. Of these, 1 was disqualified from study participation on the basis of a $36 \% \mathrm{FEV}_{1}$ at screening. The remaining 12 subjects (Table $\mathbf{1}$ ) were subsequently randomized and completed the trial.

\section{Diary Preference}

Of the 12 subjects, 7 preferred using the mobile phone diary and 5 the paper diary (Table 2). In addition, 8 of the subjects believed that the mobile diary was more accurate than the paper diary and 9 of the subjects believed that it helped them remember to take their medication. In comparison, 6 of the subjects believed that the paper diary helped them remember to take their medication. All 12 subjects believed that they provided honest and accurate reports during both diary conditions.

Eleven of the 12 subjects considered the mobile diary to be simple to use. The 1 subject who had difficulty using the mobile diary was an individual who had no prior experience using either a computer or a mobile phone. Despite this, study subjects were less likely to report all their data when using the mobile diary. Only 3 subjects stated that they recorded all their symptoms when using the mobile diary compared to 9 subjects for the paper diary. Similarly, only 7 subjects stated that they recorded all their PEFRs when using the mobile diary compared to 10 subjects for the paper diary. This discrepancy between perceived ease of use and actual use of the mobile diary appears to be related to timing issues. Only 7 subjects considered the mobile diary to be very quick to use. In addition, 2 subjects noted the inability to change the programmed reminder times and 2 subjects noted the inability to enter data late, following expiration of the programmed entry window for specific data as limitations of the mobile diary.

\section{Diary Data}

There was an association between data reporting and the type of diary employed (Table 2 ). The prevalence of missing diary entries was $\geq 20 \%$ in 4 subjects when using the mobile diary compared to only 1 subject when using the paper diary.

Table 1. Baseline Demographic and Clinical Information

\begin{tabular}{|c|c|c|c|c|c|c|c|}
\hline Subject & Age (yrs) & Gender & Race & Previous Experience Using Diaries & ICS Use at Baseline & Percent $\mathrm{FEV}_{1}$ at Baseline & Initial Diary \\
\hline 1 & 61 & Male & Caucasian & Yes & Yes & 59 & Paper \\
\hline 2 & 41 & Female & Caucasian & Yes & No & 90 & Paper \\
\hline 3 & 36 & Female & Caucasian & No & Yes & 96 & Paper \\
\hline 4 & 44 & Female & African American & Yes & No & 93 & Paper \\
\hline 5 & 43 & Female & Caucasian & Yes & No & 86 & Paper \\
\hline 6 & 43 & Male & Caucasian & No & No & 68 & Mobile \\
\hline 7 & 19 & Male & Caucasian & Yes & No & 84 & Mobile \\
\hline 8 & 21 & Male & Caucasian & Yes & No & 116 & Paper \\
\hline 9 & 45 & Male & African American & Yes & Yes & 75 & Mobile \\
\hline 10 & 23 & Female & African American & Yes & No & 90 & Mobile \\
\hline 11 & 42 & Female & Caucasian & Yes & No & 87 & Mobile \\
\hline 12 & 16 & Male & African American & Yes & No & 91 & Mobile \\
\hline
\end{tabular}


As a result, the mean prevalence of missing data points was significantly greater when using the mobile $(18 \% \pm 9 \%)$ compared to paper diary $(9 \% \pm 4 \% ; P=0.05)$. In addition, reported compliance with the treatment regimen was $8.6 \%$ lower (Mobile: $24.50 \pm 2.75$ mean total doses; Paper: 26.92 \pm 1.83 mean total doses; $P=0.05$ ). Each of these was significant in both Wilcoxon and the GLM analyses.

The following measures were significantly different in either Wilcoxon or GLM analyses but not both and thus should be considered as trends to be confirmed by future study. Reported use of rescue medication was $40 \%$ lower when using the mobile $(4.42 \pm 5.50$ doses $)$ compared to paper (7.33 \pm 6.84 doses; $P=0.03$ [GLM]) diary. In addition, there were more missing PEFRs (Mobile: $5.25 \pm 2.38$; Paper: $2.92 \pm 0.24 ; P=0.01[\mathrm{GLM}])$ and mean total symptom score was lower (Mobile: $0.50 \pm 0.39$; Paper: $0.94 \pm 0.78 ; P=0.03$ [Wilcoxon]) when using the mobile diary.

Reported PEFRs (Mobile: $439.77 \pm 89.81 \mathrm{~L} / \mathrm{min}$; Paper: $438.69 \pm 90.37 \mathrm{~L} / \mathrm{min}$ ), mean daytime symptom score (Mobile: $0.21 \pm 0.17$; Paper: $0.28 \pm 0.29)$, mean nighttime symptom score (Mobile: $0.36 \pm 0.35$; Paper: $1.57 \pm 0.51$ ), and total number of nighttime awakenings due to asthma (Mobile: $0.25 \pm 0.62$; Paper: $0.42 \pm 0.90$ ) were not significantly different between diary types when tested by either Wilcoxon or GLM analyses.

\section{Pulmonary Function Data}

Spirometry performed at the end of each treatment period suggests that, if anything, pulmonary functions were better when the subjects used the mobile diary. Overall, FEV (Fig. $^{2}$ 3 ) and $\mathrm{FEV}_{1} \%$ (Table 2 ) improved $\geq 1 \%$ of predicted normal in 7 out of the $12(58 \%)$ subjects during the mobile diary period compared to only 3 subjects $(25 \%)$ during the paper diary period. These differences did not reach significance by either test but power (17\%) was not sufficient to test the significance of differences as small as those observed in this sample.

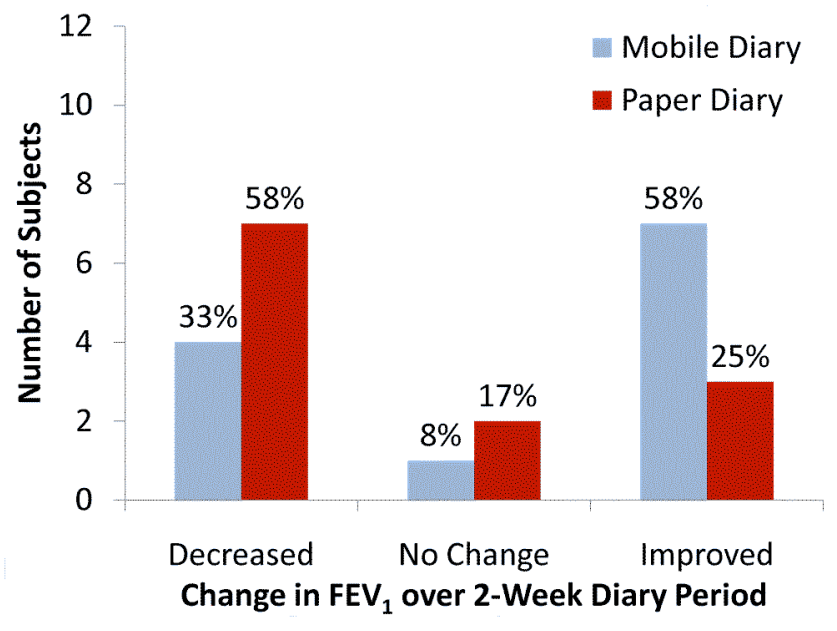

Fig. (3). Prevalence of baseline change in $\mathrm{FEV}_{1}$ over each 2 week treatment period by diary type.

\section{Ciclesonide Safety and Ease of Use}

Headache/sinus pain (3 subjects), chest pain/discomfort (2 subjects), and diarrhea ( 2 subjects) were the most commonly reported adverse events. No serious adverse events were reported and no subject discontinued the study because of an adverse event.

All 12 subjects considered the ciclesonide MDI to be very simple to learn how to use and 10 of the subjects stated that they experienced no trouble using the inhaler during the study period.

\section{DISCUSSION}

In this evaluation, there was a slight preference for use of the mobile phone diary. In addition, most subjects thought that the mobile diary was simple to use, potentially more accurate than the paper diary, and helpful in reminding them to take their medication. However, given the sample size, these findings cannot be considered definitive but instead should be used to generate questions and hypotheses for future larger scale and more completely controlled trials.

Since 9 of the 12 subjects stated that the mobile diary was helpful in reminding them to take their medication, one would expect greater treatment compliance when subjects were using the mobile dairy. It is surprising then, that the treatment compliance reported when using the mobile diary was actually $8.6 \%$ lower than that reported when using the paper diary. Given the reported symptom scores, use of rescue medication, nighttime awakenings, PEFRs, and measured $\mathrm{FEV}_{1} \mathrm{~s}$ this may not reflect lower adherence to the dosing regimen during the mobile diary phase but instead might represents erroneous reporting of compliance when using the paper diary by indicating that medication was taken when, in fact, it was taken out of schedule or not taken at all.

Reported use of rescue medication was $40 \%$ lower when using the mobile compared to paper diary. However, this finding was statistically significant in only one of the two analyses and further investigation is warranted to confirm the significance of this finding. If confirmed, it would be important to determine whether, similar to treatment compliance, this difference reflects erroneous reporting of rescue medication use or a true benefit of the mobile diary, caused by greater patient attention to their clinical status and regular use of controller medication engendered by the twice daily reminders.

Although this study was not designed to assess the accuracy of data collection using either diary type, there are suggestions that discrepancies in reported data between the 2 diary types are due to limitations of one or both diary types. Subjects stated that the inability to enter data after expiration of the predefined entry window for that data was a limitation of the mobile diary. However, after discussion with study staff, they came to recognize that the mobile diary is designed to prevent this type of data entry and the reason for this restriction. This restriction probably accounts for the higher prevalence of missing data when using the mobile diary. Several evaluations comparing electronic diaries that prevent out-of-window responses with traditional paper diaries have demonstrated similar lower completion rates for the electronic diaries [16, 19-21]. However, this reduction in completion rate may actually result in a more accurate reflection of the true data. One of the main advantages of diaries compared to recall questionnaires is obtaining responses at or near the time of the event in question, minimize the confounding effects of subject recall and subsequent events on these responses [13]. Diary responses that are not entered 
Table 2. Individual Subject Data

\begin{tabular}{|c|c|c|c|c|c|c|c|c|c|c|c|c|c|c|c|}
\hline Subject & $\begin{array}{c}\text { Diary } \\
\text { Preference }\end{array}$ & Paper & Mobile & Paper & Mobile & Paper & Mobile & Paper & Mobile & Paper & Mobile & Paper & Mobile & Paper & Mobile \\
\hline 1 & $\mathrm{P}^{*}$ & 3 & 4 & 27 & 26 & 0 & 4 & 0.0 & 0.2 & 1.1 & 1.1 & 0 & 0 & $2 \%$ & $3 \%$ \\
\hline 3 & M & 3 & 2 & 27 & 28 & 2 & 2 & 0.2 & 0.1 & 0.1 & 0.0 & 0 & 0 & $-7 \%$ & $-4 \%$ \\
\hline 4 & $\mathrm{M}$ & 1 & 3 & 29 & 27 & 6 & 0 & 0.2 & 0.3 & 0.6 & 0.2 & 0 & 0 & $-2 \%$ & $-4 \%$ \\
\hline 5 & M & 2 & 4 & 28 & 26 & 1 & 0 & 0.0 & 0.0 & 0.1 & 0.0 & 0 & 0 & $1 \%$ & $1 \%$ \\
\hline 6 & $\mathrm{P}$ & 3 & 3 & 27 & 27 & 6 & 5 & 0.4 & 0.3 & 0.1 & 0.1 & 0 & 1 & $0 \%$ & $6 \%$ \\
\hline 10 & M & 6 & 8 & 24 & 22 & 3 & 3 & 0.0 & 0.0 & 0.1 & 0.3 & 0 & 0 & $-1 \%$ & $1 \%$ \\
\hline 11 & M & 4 & 5 & 26 & 25 & 6 & 8 & 0.2 & 0.3 & 0.2 & 0.5 & 0 & 0 & $0 \%$ & $0 \%$ \\
\hline 12 & M & 3 & 9 & 27 & 21 & 18 & 0 & 0.8 & 0.0 & 0.8 & 0.5 & 0 & 0 & $-10 \%$ & $2 \%$ \\
\hline
\end{tabular}

P: preferred paper diary; ${ }^{\dagger} \mathrm{M}$ : preferred mobile diary.

contemporaneous with the event in question are subject to these same confounding effects, eliminating this advantage. Entries in paper diaries are not time stamped and are frequently not made at the time the investigator expects, potentially introducing errors into the analysis. In one study, for example, subjects were asked to record data in a paper diary at 3 specific times daily for 3 weeks. When the diaries were returned, $90 \%$ of the expected data had been entered. However, a sensor hidden in the binding of the diaries demonstrated that only $11 \%$ of these entries were made within the prescribed time frame. Furthermore, on $32 \%$ of study days the diaries were never even opened [12].

In the current trial, subjects with mild to moderate persistent asthma were approximately $90 \%$ compliant with their prescribed controller regimen over a 2 -week period. This compliance would most likely have decreased had this been a longer trial. Using pharmacy claims data a $34 \%$ initial and $25 \%$ 6-month compliance rate with ICS therapy has been reported for subjects not actively participating in a study protocol [22]. Although using a diary with an intrinsic electronic reminder system did not appear to improve compliance compared to using a paper diary in the current evaluation, this comparison depends upon paper diary entries that, as previously noted, may be neither timely nor accurately recorded. A previous evaluation comparing paper diary responses with actual ICS use, as determined by an electronic monitoring device, found that paper diary responses consistently overstated actual ICS use [23]. Similar to the current evaluation, patients with asthma who were evaluating another mobile phone-based system stated that the electronic reminder was helpful in remembering to take their medication and that they missed this reminder once participation in the trial ended [14]. Moreover, use of a mobile phone-based reminder system to improve compliance has been shown to be effective in other disorders [24]. This reminder activity warrants further investigation in the routine clinical management of patients with persistent asthma.

Finally, it is important to consider the limitations of the current evaluation. This was a small pilot study designed to assess acceptance and feasibility of using a mobile phonebased system to electronically capture clinical information in subjects with mild to moderate persistent asthma. It was not designed to assess the accuracy of the data recorded in the diaries nor powered to detected significant differences between the two diary types in adherence or functional status outcomes. As such, it does not provide definitive answers. However, despite an estimated worldwide prevalence of 2.7 billion mobile phones [17], few data exist on using this technology for electronic data collection, especially in patients with asthma. Previous evaluations of the use of mobile phone-based diary systems in patients with asthma by Anhoj and Cleland assessed 12 and 10 subjects, respectively [14, 25]. The 12 subjects described in the current evaluation substantially augment this knowledge base, confirming the feasibility and potential value of this technique and hopefully stimulating larger, more definitive randomized controlled clinical trials. Current data on subject acceptance of electronic data capture devices is also limited and contradictory. For example, in one crossover evaluation, $85 \%$ of subjects preferred a traditional paper diary over an interactive voice response system [15] whereas in another, $59 \%$ of subjects preferred a handheld computer system over a traditional paper diary [21]. In the current evaluation, $58 \%$ of subjects preferred the mobile phone-based diary, lending support to the concept that subjects will accept and ultimately may prefer to use electronic data capture devices.

\section{CONCLUSIONS}

In this pilot evaluation, the VOCEL ${ }^{\circledR}$ Mobile Diary was considered to be easy to use and was preferred by slightly 
more subjects than the paper diary. Despite this, there were more missing data entries when recording with the mobile diary, most likely related to the strictly enforced time periods for these entries. Whether the additional entries in the paper diary are meaningful however, cannot be determined since there were no objective measures of either the time or the accuracy of these entries. Nonetheless, the apparent discrepancy between diary reports of treatment compliance and asthma symptoms, need for rescue medication, and pulmonary function tests suggests that these additional entries may not be completely reliable.

The ciclesonide MDI was safe, effective, and easy to use. Large, randomized, controlled clinical trials are warranted to confirm these findings and further evaluate the effect of diary type on recorded responses.

\begin{tabular}{|c|c|}
\hline \multicolumn{2}{|c|}{ ABBREVIATIONS } \\
\hline $\mathrm{FEV}_{1}$ & $=$ Forced Expiratory Volume in 1 second \\
\hline GLM & $=$ General Linear Models \\
\hline HFA & $=$ Hydrofluoroalkane \\
\hline ICS & $=$ Inhaled corticosteroid \\
\hline MDI & $=$ Metered dose inhaler \\
\hline PEFR & $=$ Peak expiratory flow rate \\
\hline
\end{tabular}

Sanofi-Aventis and VOCEL provided funding for this study. Sanofi-Aventis provided the inhaled study medications and suggested the dose of ciclesonide. VOCEL assisted in the design of the electronic diary and provided the electronic units. In addition, VOCEL provided funding for manuscript preparation. Neither Sanofi-Aventis nor VOCEL had a role in the analysis or interpretation of data, or in the contents of the manuscript.

We thank Gerald Barber, MD who provided medical writing services to the authors. Dr. Barber was compensated for these services with funds provided by VOCEL, Inc.

\section{CONFLICT OF INTEREST}

\section{Eli O. Meltzer:}

Grant/Research Support: Alcon, Altana/Nycomed, Amgen, AstraZeneca, Boehringer Ingelheim, Capnia, Critical Therapeutics, Genentech, GlaxoSmithKline, Medicinova, MedPointe, Merck, Novartis, Sanofi-Aventis, Schering-Plough, VOCEL, Wyeth.

Consultant: Abbott, Alcon, Allux, Altana/Nycomed, Amgen, APT, AstraZeneca, Capnia, Critical Therapeutics, Dey, Genentech, GlaxoSmithKline, Greer, Inspire, MedPointe, Merck, Novartis, Pfizer, Sanofi-Aventis, Schering-Plough, Sepracor, Verus, Wyeth.

Norma Kelley and Melbourne F Hovell: None.

\section{SUPPORTIVE/SUPPLEMENTARY MATERIAL}

Post_Study_Questions.pdf. Questionaire used at the conclusion of the second study period. This supplementary material can be viewed at www.bentham.org/open/tormj.

\section{REFERENCES}

[1] National Asthma Education and Prevention Program. Guidelines for the diagnosis and management of asthma (EPR-3). National Institutes of Health [monograph on the Internet]. 28 Aug 2007 [accessed 19 Oct 2007]. Available from: http://www.nhlbi.nih.gov/guidelines/asthma/

[2] Global Initiative For Asthma (GINA). Global Strategy for Asthma Management and Prevention. Global Initiative For Asthma [monograph on the Internet]. 2006 [accessed 19 Oct 2007]. Available from: http://www.ginasthma.org

[3] Boulet LP, Drollmann A, Magyar P, et al. Comparative efficacy of once-daily ciclesonide and budesonide in the treatment of persistent asthma. Respir Med 2006; 100: 785-94.

[4] Adachi M, Ishihara K, Inoue H, et al. Efficacy and safety of inhaled ciclesonide compared with chlorofluorocarbon beclomethasone dipropionate in adults with moderate to severe persistent asthma. Respirology 2007; 12: 573-80.

[5] Hansel TT, Benezet O, Kafe H, et al. A multinational, 12-week, randomized study comparing the efficacy and tolerability of ciclesonide and budesonide in patients with asthma. Clin Ther 2006; 28 : 906-20.

[6] Dyer MJ, Halpin DM, Stein K. Inhaled ciclesonide vs inhaled budesonide or inhaled beclomethasone or inhaled fluticasone for chronic asthma in adults: a systematic review. BMC Fam Pract 2006; 7: 34 .

[7] Buhl R, Vinkler I, Magyar P, et al. Comparable efficacy of ciclesonide once daily vs fluticasone propionate twice daily in asthma. Pulm Pharmacol Ther 2006; 19: 404-12.

[8] Niphadkar P, Jagannath K, Joshi JM, et al. Comparison of the efficacy of ciclesonide 160 microg QD and budesonide 200 microg BID in adults with persistent asthma: a phase III, randomized, double-dummy, open-label study. Clin Ther 2005; 27: 1752-63.

[9] Cowie RL, Boulet LP, Keith PK, Scott-Wilson CA, House KW, Dorinsky PM. Tolerability of a salmeterol xinafoate/fluticasone propionate hydrofluoroalkane metered-dose inhaler in adolescent and adult patients with persistent asthma: a 52-week, open-label, stratified, parallel-group, multicenter study. Clin Ther 2007; 29: 1390-402.

[10] Murphy K, Noonan M, Silkoff PE, Uryniak T. A 12-week, multicenter, randomized, partially blinded, active-controlled, parallelgroup study of budesonide inhalation suspension in adolescents and adults with moderate to severe persistent asthma previously receiving inhaled corticosteroids with a metered-dose or dry powder inhaler. Clin Ther 2007; 29: 1013-26.

[11] Trautmann M, Banik N, Tews JT, Jorres RA, Nowak D. Efficacy of the combination of fluticasone propionate and salmeterol in patients with moderate persistent asthma within a "real-life" setting. Eur J Med Res 2007; 12: 255-63.

[12] Stone AA, Shiffman S, Schwartz JE, Broderick JE, Hufford MR. Patient non-compliance with paper diaries. BMJ 2002; 324: 11934.

[13] Piasecki TM, Hufford MR, Solhan M, Trull TJ. Assessing clients in their natural environments with electronic diaries: rationale, benefits, limitations, and barriers. Psychol Assess 2007; 19: 25-43.

[14] Anhoj J, Moldrup C. Feasibility of collecting diary data from asthma patients through mobile phones and SMS (short message service): response rate analysis and focus group evaluation from a pilot study. J Med Internet Res [serial on the Internet]. 2004 [accessed 7 Aug 2008]; 6: e42. Available from: http://www.jmir.org/2004/4/e42/

[15] Weiler K, Christ AM, Woodworth GG, Weiler RL, Weiler JM. Quality of patient-reported outcome data captured using paper and interactive voice response diaries in an allergic rhinitis study: is electronic data capture really better? Ann Allergy Asthma Immunol 2004; 92: 335-9.

[16] Nyholm D, Kowalski J, Aquilonius SM. Wireless real-time electronic data capture for self-assessment of motor function and quality of life in Parkinson's disease. Mov Disord 2004; 19: 446-51.

[17] Cox WM, Alm R. The best of all worlds: globalizing the knowledge economy. Federal Reserve Bank of Dallas 2006 Annual Report. Federal Reserve Bank of Dallas [monograph on the Internet]. 2006 [accessed 25 Jun 2008]. Available from: http://www.dallasfed.org/fed/annual/2006/ar06.pdf

[18] National Heart, Lung, and Blood Institute. Practical guide for the diagnosis and management of asthma. NIH Publication 97-4053. Published October 1997. 
[19] Gaertner J, Elsner F, Pollmann-Dahmen K, Radbruch L, Sabatowski R. Electronic pain diary: a randomized crossover study. J Pain Symptom Manage 2004; 28: 259-67.

[20] Lauritsen K, Degl' Innocenti A, Hendel L, et al. Symptom recording in a randomised clinical trial: paper diaries $v s$ electronic or telephone data capture. Control Clin Trials 2004; 25: 585-97.

[21] Tiplady B, Crompton GK, Dewar MH, et al. The use of electronic diaries in respiratory studies. Drug Infor J 1997; 31: 759-64.

[22] Jones C, Santanello NC, Boccuzzi SJ, Wogen J, Strub P, Nelsen LM. Adherence to prescribed treatment for asthma: evidence from pharmacy benefits data. J Asthma 2003; 40: 93-101.
Berg J, Dunbar-Jacob J, Rohay JM. Compliance with inhaled medications: the relationship between diary and electronic monitor. Ann Behav Med 1998; 20: 36-8.

[24] Vilella A, Bayas JM, Diaz MT, et al. The role of mobile phones in improving vaccination rates in travelers. Prev Med 2004; 38: 5039.

[25] Cleland J, Caldow J, Ryan D. A qualitative study of the attitudes of patients and staff to the use of mobile phone technology for recording and gathering asthma data. J Telemed Telecare 2007; 13: 85-9.

Received: July 2, 2008

Revised: August 6, 2008

Accepted: August 8, 2008

(C) Meltzer et al.; Licensee Bentham Open.

This is an open access article licensed under the terms of the Creative Commons Attribution Non-Commercial License (http://creativecommons.org/licenses/by$\mathrm{nc} / 3.0 /$ ) which permits unrestricted, non-commercial use, distribution and reproduction in any medium, provided the work is properly cited. 